Aus der Lichtabteilung des Irankenhauses St. Göran in Stockholm (Oberarzt Dr. Magnus Möller).

\title{
Ein neuer mechanischer Kompressor bei der Lichtbehandlung nach Finsen.
}

\author{
Von \\ F. Sandman,
}

Assistent.

(Hiezu Taf. IV.)

Bei der weiteren Ausbildung seiner Lichtmethode gegen Lupus vulgaris fand $\mathrm{F}$ insen, daß bei dieser Behandlung die Blutleere des bestrahlten Hautteiles ein wichtiges Detail sei; auf einfache Weise wurde dies dadurch erreicht, daß eine Linse gegen die Haut gedrückt wurde. Anfangs glaubte er, daß sich ein gutes Resultat dadurch erreichen ließe, daß die DruckInse mit Gummibändern befestigt wurde, welche dann den notwendigen Druck ausüben sollten; aber er fand bald heraus, daß dies eine recht unvollkommene Weise sei. Einesteils wird nämlich die Spannung in den vier Bändern bald ungleich und die Linse schief gestellt; andernteils ist die Kraft der Gummibänder nicht hinreichend genug. Nach und nach ging er fast ausschließlich zur Anwendung manueller Kraft über. Jede Linse sollte unter der Seance von einer Pflegerin gehandhabt werden, welche dafür die Verantwortung übernahm, daß die Linse richtig eingestellt sei und ein unausgesetzter, gleichmäßiger Druck erreicht werde, genügend stark, $\mathrm{nm}$ die betreffende Hautpartie blutleer zu machen. Diese Anforderung verteuert natürlich die Behandlung; außerdem bleibt es fraglich, ob die Anwendung menschlicher Kraft in diesem Falle das richtige sei. Man verlangt, daß eine Person länger als eine Stunde einen ununterbrochenen und starken Druck aus- 
üben soll, lediglich durch Spannung der Armmuskeln; dies dürfte geradezu unmöglich sein.

Man hat deshab versucht die manuelle Kraft durch Apparate zu ersetzen, abgesehen davon, daß man sich mitunter mit den oben besprochenen Gummibändern begnügt hat. So haben Mari ${ }^{1}$ ) und $\mathrm{Mez}$ er et t $\mathrm{e}^{2}$ ) jeder einen Apparat für mechanische Kompression beschrieben, die bei kleineren Lampen für die Behandlung von nur je einer Person gebraucht werden. W i c h $\mathrm{m} \mathrm{a} n \mathrm{n}^{3}$ ) beschreibt eine Anordnung bei der Finsen-Reyn-Lampe, welche manuellen Druck unnötig macht. Schließlich hat A. J u n g m a n $\mathrm{n}^{4}$ ) von der Heilstätte für Lupuskranke in Wien eine automatische Drucklinse konstruiert, welche an der großen 4-armigen Finsenlampe appliziert wird. Er fixiert zuerst jenen Körperteil, der behandelt werden soll; in den meisten Fällen ist dies ja der Kopf und geschieht dies mittels einer Stütze, welche sowohl den unteren Teil als auch den der Linse entgegengesetzten Teil des Kopfes umfaßt. Die Drucklinse ist durch drei zylindrisch gewundene Druckfederspiralen, welche an drei Achsen, die mit Schraubengewinde versehen sind, laufen, an den Tubus befestigt. Diese Drucklinse wird nun gegen die Hautpartie eingestellt, auf welcher der notwendige Druck erreicht wird, teils dadurch, daß der untere Teil des Tubus durch einen Zahntrieb gesenkt wird, teils dadurch, daß die Spannung in den drei vorher besprochenen Spiralen vergrößert wird.

Der Übelstand bei dieser Anordnung ist der, daß nicht alle Tuben mit diesem Zahntrieb versehen sind; auch müssen recht komplizierte Veränderungen am Tubus zwecks Befestigung der Drucklinse vorgenommen, sowie solche selbst. passend für die Anordnung, angeschafft werden. Schließlich soll man ja nicht gerne die Länge des Tubus variieren. Im übrigen erscheint der Apparat wohl ausgedacht und "wird auch seit längerer Zeit mit vorzüglichem Erfolge angewandt". Lesser berichtet in einer Diskussion der Berliner Dermatologischen Gesellschaft, ${ }^{5}$ ) daß die Behandlung bei einer ganzen Reihe

1) Archives d'electricité médicale. 1904.

2) Annales de Dermatologie et de Syphiligraphie. 1905.

3) Deutsche medizin. Wochenschrift. 1905. Nr. 50.

4) Wiener klinische Wochenschrift. 1906. Nr. 28.

5) Dermatologische Zeitschrift. 1907 Seite. 4l. 
von Stellen mit diesem mechanischen Druckglas ganz gut geht. - Schon bevor diese letzte Mitteilung vorlag, wurde in der von Dr. M öller geleiteten Abteilung des Krankenhauses St. Göran in Stockholm ein Apparat angewandt, welcher denselben Zweck wie die oben beschriebenen verfolgt. Nach meinen Anweisungen hatte nämlich Herr A. Nyström, bei Firma Alb. Stille angestellt, einen mechanischen Drucklinsenapparat angefertigt. Aus der Abbildung sind die Hauptteile deutlich zu ersehen. Dieselben bestehen aus einem rechtwinkelig gebogenen Stahlrohre $a$, welches vermittels zwei Klammern an den Behandlungstisch angeschraubt wird. An dem vertikalen Teile des Rohres $a$ ist unten die Stütze für den Kopf befestigt, oben die Führungsstange für die Drucklinse. Die Beschaffenheit der Kopfstütze ersieht man am besten aus der Abbildung. Teile:

Die Befestigung der Drucklinse geschieht durch folgende

1. Ein Arm $c$, welcher an der vertikalen Stange $a$ aufund abwärts bewegt und auch um dieselbe rotiert werden kann. Durch die Schraube $d$ wird der Arm $e$ fixiert.

2. Ein auf $c$ laufender Teil $g$, der durch ein Gelenksystem $f$ in jeder gewünschten Lage festgehalten werden kann. Der Teil $g$ kann sowohl auf- und abwärts geschoben, als auch um seine eigene Achse gedreht werden. Durch die Schraubeneinrichtung $h$ kann die Linse ebenfalls in der Richtung ihrer Längsachse verschoben werden.

An dem Teile $g$ wird nun die Drucklinse derart angebracht, $\mathrm{da} \mathfrak{B}$ einer der Arme in die Öse $i$ geschoben wird, worauf man die Schraube $k$ einsetzt und anzieht.

Um zu verhindern, daß sich der Druck der Linse verringert, wenn das Hautgewebe zusammengepreßt und blutleer wird, so wurde in den Teil $g$ eine Spiralfeder eingesetzt, welche bewirkt, daß die Linse beim Einsinken der Haut nachfolgt.

Die Einstellung der Drucklinse geschieht derart, daf der zu behandelnde Körperteil (gewöhnlich der Kopf) auf die Stütze placiert wird und zwar so hoch, daß die zu beleuchtende Partie ein wenig innerhalb des Fokus zu liegen kommt. Nachher läßt man die Schrauben $d$ und $f$ nach, placiert die Drucklinse auf der Haut und gibt Acht, daß die Linse senkrecht zur Richtung 
der Strahlen steht, welches aus der Lage des Spiegelbildes ersichtlich ist. Ist dies erreicht, so fixiert man die Linse durch Anziehen der Schrauben $d$ und $f$; der gewünschte Druck wird nun durch die Schraube $h$ erlangt. Die Schraube $h$ verändert die Lage der Linse nur insofern, als dieselbe in der Richtung der Strahlen verschoben wird. Besonders hervorheben möehte ich den Vorteil, daß, wenn beim Placieren der Linse die Schrauben $d$ und $f$ gelockert sind, eine erhöhte Beweglichkeit und Leichtigkeit der Einstellung gewonnen wird.

Die Vorteile dieses Apparates sind folgende:

1. Ein gleichförmiger, federnder Druck von jeder gewünschten Stärke kann erreicht werden.

2. Der Apparat ist bei einiger Übung leicht einzustellen, gewöhnlich innerhalb 1-3 Min. Einmal eingestellt braucht der selbe kaum beaufsichtigt zu werden während der Seance.

3. Der zu behandelnde Körperteil (Kopf) wird sicher fixiert.

4. Man braucht die Länge des Tubus nicht zu verändern.

5. Keinerlei Umänderungen sind an den Tuben oder Drucklinsen nötig, wenn diese die gebräuchlichen Typen sind. Der Apparat ist relativ billig.

Meinem Chef, Herrn Doktor Magnus Möller, durch dessen Wohlwollen und Interesse es mir ermöglicht wurde, den oben beschriebenen Apparat auszuführen und zu erproben, erlaube ich mir auch an dieser Stelle meinen herzlichsten Dank auszudrücken.

Da Dr. Sandman nichts über den Behandlungserfolg bei Anwendung des von ihm konstruierten Kompressors erwähnt hat, so erlaube ich mir hier das Urteil auszusprechen, daß dieser ganz vorzüglich ist. Die Reaktionen werden immer sehr scharf umschrieben und möglichst intensiv. Dies war ja auch bei der absolut genauen und gleichmäßigen Fixierung, die der Apparat mit sich bringt, zu erwarten. Die Patienten haben in der Regel den Apparat gern, ausnahmsweise kann der eine oder andere nach etwa einer halben Stunde durch die unnachgebliche Fixierung müde werden und wünscht für einige Minuten losgeschraubt zu werden. Sonst braucht nach Einstellung des Druckglases der Apparat während der ganzen Seance kaum beaufsichtigt $\mathrm{zu}$ werden. Nach Anschaffung von zwei Apparaten haben wir das Pflegerinnenpersonal um eine vermindern können. Es ist mir eine angenehme Pflicht, den Sandmanschen Kompressor warm zu empfehlen.

Magnus Möller. 


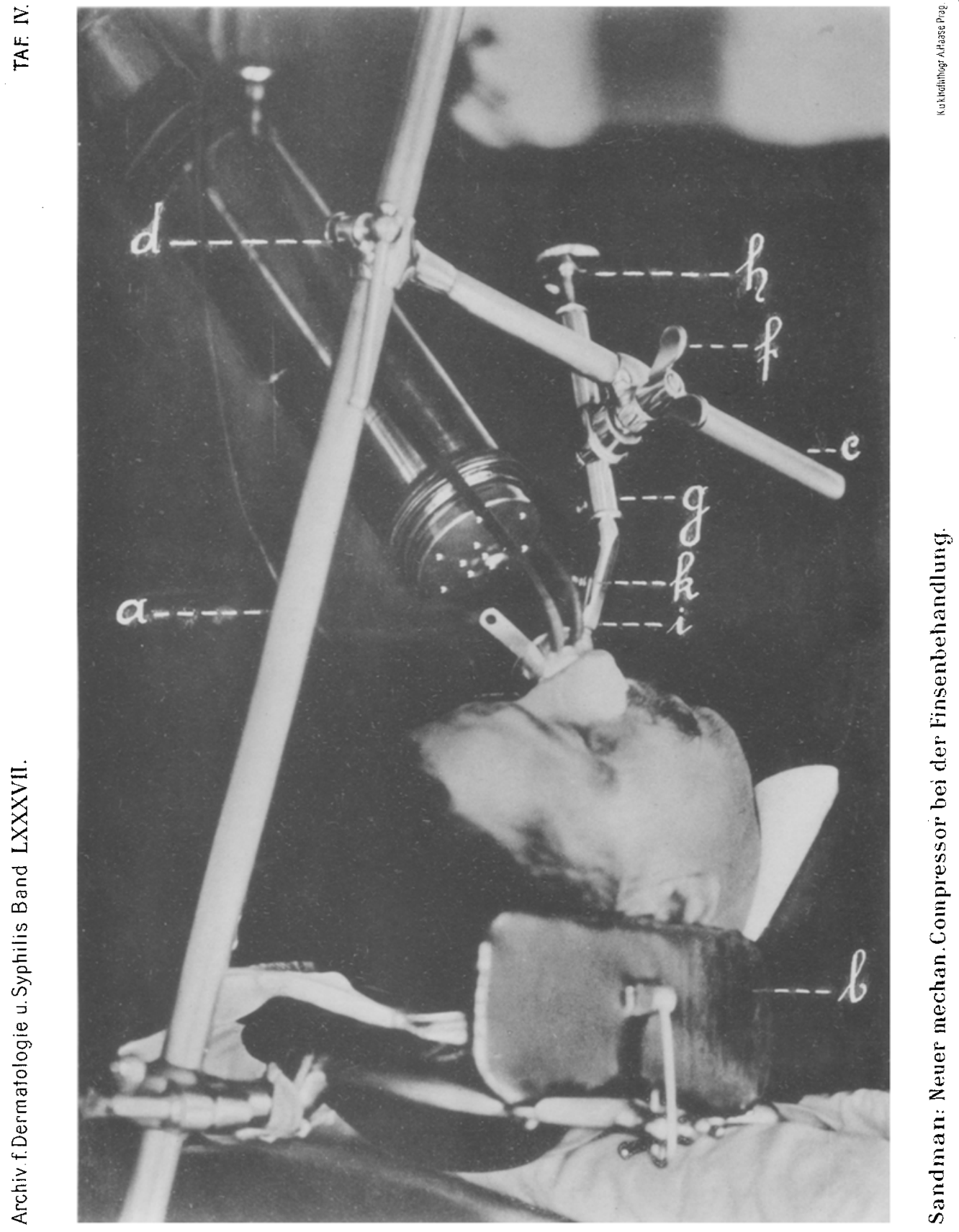

